H Almuhtaseb ${ }^{1,2}$ and A Lotery ${ }^{1,2}$

${ }^{1}$ Eye Unit, University Hospital Southampton NHS

Foundation Trust, Southampton, UK

${ }^{2}$ Clinical and Experimental Sciences, University of

Southampton, Southampton, UK

E-mail: a.j.lotery@soton.ac.uk

Eye (2018) 32, 479-481; doi:10.1038/eye.2017.212;

published online 20 October 2017

\section{Sir, \\ Simple fixation and removal of dacryocystorhinostomy tube}

The use of silicone tubes as an adjunct to dacryocystorhinostomy (DCR) surgery is common place although the evidence base for their use remains weak. ${ }^{1}$ The tubes are usually fixed intranasally with a variety of techniques, aiming to improve the stability and ocular comfort. It is not known whether different ways of securing the tubes affects the DCR outcome.

Most extubation techniques entail cutting the stents at their inter-punctal portion and any endonasal fixation sutures used, and then pulling the tubes out from the nose, which sometimes requires endoscopic assistance.

We read with great interest the DCR stent fixation and removal technique by Galea et al, ${ }^{2}$ which involves insertion of a bicanalicular silicone tube tied with two 6/0 silk sutures, and removal by cutting the loop of the tube close to the lower punctum, followed by pulling the upper cut end of the tube laterally through the upper canaliculus to externalize the rest of the tube and sutures.

Extubation via medial canthus has been documented previously. Crawford ${ }^{3}$ described removal of silicone tubing (fixed with intraluminal $8 / 0$ silk suture) by grasping the tube below the upper punctum, cutting the loop just above the lower punctum and pulling it out. Dressner ${ }^{4}$ also advocated a relatively similar fixation and removal procedure.

Recently we reported our technique for DCR tube fixation using a short silicone sleeve and external removal via medial canthus. ${ }^{5}$ The fixation is carried out by passing the bodkins of the O'Donohue tubes through a short (2 $\mathrm{mm}$ ) silicone (Watzke) sleeve in a crossed manner, followed by partial retrieval of the tubes through the DCR incision. The tightness of the tube is then tested and adjusted by pulling the loop of the tube from the medial canthus (Figure 1a and b).

The tube is simply removed externally by grasping it at the medial canthus with non-toothed forceps and firmly pulling laterally in a single movement (Figure 1c). This obviates the need of any intranasal manipulation or tube cutting. The short sleeve segment rests within the nose before finding its own way out. The patient must be warned to expect a momentary discomfort during removal which quickly passes. In our retrospective analysis of 166 cases of external DCRs using this method, none had retained tube/sleeve material or tube-related canalicular/punctal damage.

A main concern with these extubation techniques via medial canthus is the risk of subtle epithelial injury to the area of the common opening with potential subsequent membrane formation. Although we did not encounter any silicone sleeve related issues, further studies with endoscopic evaluation may unambiguously address this concern. Galea et al reported retained tube material in $7.5 \%$ of the cases, ${ }^{1}$ which may be related to removal of both ends (still joined by sutures) through upper canalicular system.

In conclusion, our very low rate of tubes related complications in terms of both tube stability and patient comfort and the speed and simplicity of adjustment and removal encourages us to promote the use of a $2 \mathrm{~mm}$ long silicone sleeve to join the ends of a stent during external DCRs. Its main advantage is the quick and easy removal technique.

\section{Conflict of interest}

The authors declare no conflict of interest.

\section{References}

1 Feng Y, Ca J, Zhang J, Han X. A meta-analysis of primary dacryocystorhinostomy with and without silicone intubation. Can J Opthalmol 2011; 46(6): 5217.

2 Galea M, Shams F, Saxby E, Newcott E, Chadha V, Kemp E. Extubation following dacryocystorhinostomy: an alternative technique. Eye 2017; 31(7): 982-984.
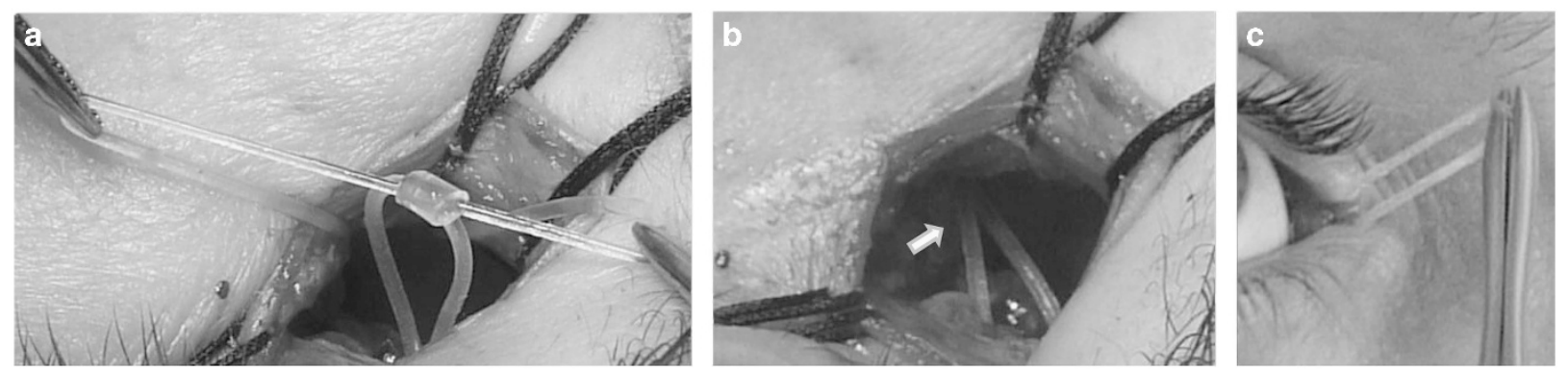

Figure 1 (a) Partial retrieval of the tube through DCR incision and adjustment of the tension. Note ends already passed through the sleeve in a crossed fashion. (b) Sleeve and stent position post-adjustment (arrow). (c) Removal of the tube via medial canthus by pulling the loop laterally with a Moorefield forceps (without any cutting). 
3 Crawford JS. Lacrimal intubation set with suture in the lumen. Ophthal Plast Reconstr Surg 1988; 4(4): 249-250.

4 Dresner SC. Silicone intubation without intranasal fixation for treatment of congenital nasolacrimal duct obstruction. Am J Ophthalmol 1995; 119(6): 816-817.

5 Vahdani K, Thaller VT, Albanese G. Easy

dacryocystorhinostomy stent fixation and removal: use of a silicone sleeve and external stent removal from the medial canthus. Orbit 2017; 36(3): 175-177.
K Vahdani ${ }^{1}$, G Albanese ${ }^{2}$ and VT Thaller ${ }^{3}$

${ }^{1}$ Bristol Eye Hospital, Bristol, UK

${ }^{2}$ Department of Ophthalmology, Nottingham University Hospitals NHS Trust, Nottingham, UK

${ }^{3}$ Royal Eye Infirmary, Derriford Hospital, Plymouth, UK E-mail: kaveh.vahdani@nhs.net

Eye (2018) 32, 481-482; doi:10.1038/eye.2017.215; published online 13 October 2017 